\title{
Production comparée de zooplancton en bassins fertilisés avec la fiente de volaille et la bouse de vache
}

\author{
Hyppolite AGADJIHOUEDE ${ }^{1 *}$, Clément Agossou BONOU ${ }^{2}$, Antoine CHIKOU ${ }^{1}$ et \\ Philippe LALEYE ${ }^{1}$ \\ ${ }^{1}$ Laboratoire d'Hydrobiologie et d'Aquaculture / Faculté des Sciences Agronomiques/Université d'Abomey- \\ Calavi, 01 BP 526 Cotonou, Bénin. \\ ${ }^{2}$ Laboratoire de Recherche en Biologie Appliquée / Ecole Polytechnique d'Abomey-Calavi/ Université \\ d'Abomey-Calavi. 01 BP 2009 Cotonou, Bénin. \\ *Auteur correspondant, E-mail : agadjihouede@yahoo.fr, Tél : (00229) 97265704
}

\section{RESUME}

En vue d'identifier le meilleur fertilisant qui pourra être utilisé pour la production intensive du zooplancton en milieu contrôlé, les essais de production du zooplancton en bassins fertilisés à la fiente de volaille et à la bouse de vache sont réalisés et les résultats comparés. Le dispositif expérimental est constitué de 06 bassins dont 02 pour la fiente, 02 pour la bouse et 02 pour le témoin. Après fertilisation, le zooplancton est ensemencé le même jour avec 1 ind. $\mathrm{L}^{-1}$ de nauplius de copépodes, 2 ind. $\mathrm{L}^{-1}$ de copépodes, 3 ind. $\mathrm{L}^{-1}$ de rotifères et 1 ind. $\mathrm{L}^{-1}$ de cladocères par bassin. Un suivi des paramètres nutritifs et biologiques des milieux de production a été réalisé tous les 2 jours pendant 30 jours. Les paramètres physico-chimiques ont très peu varié dans les bassins. La température moyenne est de $29,6 \pm 0,1^{\circ} \mathrm{C}$; l'oxygène dissous moyen est de $6,8 \pm 0,7 \mathrm{mg} \cdot \mathrm{L}^{-1}$ et le $\mathrm{pH}$ moyen est de $9,1 \pm 0,1$. Les forts taux de nitrates $\left(4,55 \mathrm{mg} \cdot \mathrm{L}^{-1}\right)$ et de phosphates $\left(19,79 \mathrm{mg} . \mathrm{L}^{-1}\right)$ sont obtenus avec la fiente de volaille. La densité du zooplancton qui est de 7 ind. $\mathrm{L}^{-1}$ dans tous les bassins est passée à 2022 ind. $\mathrm{L}^{-1}$ (maximum) en 6 jours dans les bassins fertilisés à la bouse de vache, à 3046 ind. $\mathrm{L}^{-1}$ (maximum) en 6 jours dans les bassins fertilisés à la fiente de volaille et à 772 ind. $\mathrm{L}^{-1}$ (maximum) en 21 jours dans les bassins témoins. Les résultats obtenus ont montré que la fiente de volaille libère plus vite les sels nutritifs dans l'eau et donne la meilleure biomasse phytoplanctonique (taux de chlorophylle-a plus élevés ; $\mathrm{p}<0,05$ ). La plus forte densité du zooplancton (3046 ind. $\left.\mathrm{L}^{-1}\right)$, la production journalière la plus élevée $\left(41\right.$ ind. $\left.\mathrm{L}^{-1} . \mathrm{J}^{-1}\right)$ et le meilleur taux d'accroissement $(\mathrm{Kr}, 24$ heures $=0,7)$ est obtenu dans les bassins à fiente de volaille. Ainsi, la fiente de volaille se révèle comme le meilleur fertilisant pour la production du zooplancton.

(C) 2010 International Formulae Group. All rights reserved

Mots clés : Culture, zooplancton, bassins, fiente de volaille, bouse de vache.

\section{INTRODUCTION}

L'utilisation du zooplancton en aquaculture est d'un grand intérêt (Bonou, 1990 ; Dabbadié, 1996), en tant que source alimentaire des larves de poissons à faibles réserves vitellines. Le zooplancton est un aliment plus performant qu'un aliment artificiel (Awaïss et al., 1993). En effet, le zooplancton paraît le mieux proportionnel de par sa taille à l'ouverture buccale de certaines larves encore trop petites (Bonou et al., 1991). De même, le zooplancton est utilisé comme complément alimentaire en système semiintensif lors de pré grossissement ou de 
grossissement des poissons (Bonou, 1990). Ainsi, pour le développement de l'aquaculture, la production intensive de ces microorganismes planctoniques est nécessaire. C'est à cet effet que des techniques sont mises en place surtout en Asie et en Europe à partir des travaux de recherche.

En Afrique tropicale, les techniques de production généralement utilisées sont basées sur la fertilisation des étangs et des bassins de pisciculture avec les fumures organiques et inorganiques (Broussard et al., 1983 ; Vincke et Philippart, 1984 ; James et al., 1989 ; Bonou, 1990 ; Rukera Tabaro et al., 2005). Les fumures organiques les plus utilisées sont les fientes de volaille, les fumures de vache, de porc et d'autres techniques associées (Lacroix, 2004).

La présente étude vise à déterminer le meilleur fertilisant à utiliser pour une production intensive du zooplancton. Ainsi, l'aptitude de la bouse de vache et de la fiente de volaille à favoriser une bonne production du zooplancton est comparée et le meilleur fertilisant en est déduit.

\section{MATERIEL ET METHODES}

La production du zooplancton est réalisée dans 06 bassins de $1 \mathrm{~m}^{3}$ remplis d'eau à $0,30 \mathrm{~m}$ de hauteur. Pour ce remplissage, l'eau de robinet et l'eau d'étang filtrée au moyen d'un filet de $50 \mu \mathrm{m}$ de maille sont utilisées à volume égal. Ensuite, 02 bassins sont fertilisés avec la fiente de volaille (race : Isa brown), 02 avec la bouse de vache à raison de $200 \mathrm{~g}$ de poids sec et 02 bassins non fertilisés (temoins). Le même jour de la fertilisation, les bassins sont ensemencés de zooplancton récoltés par filtration de l'eau d'étang d'élevage de Tilapia au moyen du filet de $50 \mu \mathrm{m}$. La densité de mise en charge est de 7 ind. $\mathrm{L}^{-1}$ (3 ind. $\mathrm{L}^{-1}$ de copépodes; 3 ind. $\mathrm{L}^{-1}$ de rotifères et 1 ind. $\mathrm{L}^{-1}$ de cladocères). Au total, trois traitements sont appliqués et répétés une fois. Il s'agit des bassins fertilisés avec de la bouse de vache (BV), des bassins fertilisés avec de la fiente de volaille (FV) et des bassins non fertilisés ou bassins témoins (BT).
Le pH, l'oxygène dissous et la température de l'eau sont mesurés dans chaque bassin tous les 2 jours avec le pHTestr 10 et l'oxythermomètre VOLTCRAFT à 0,01 mg. $\mathrm{L}^{-1}$ et $0,1^{\circ} \mathrm{C}$ près.

Les teneurs en sels nutritifs (nitrates et les phosphates) sont dosés par la méthode colorimétrique Nitra-Ver 3 et Phos ver 3 (Color Disc Method 24609-00, Hach Company World Headquarters, USA) et les particules nutritives en suspension (algues et autres micro-organismes) sont évaluées par la production de la chlorophylle-a exprimée en mg. $\mathrm{L}^{-1}$. Le dosage de la chlorophylle-a est réalisé en utilisant la méthode de Lorenzen (1967) modifiée, proposée par le Centre d'Etude sur les Systèmes Aquatiques Continentaux (C.E.S.A.C) de l'Université de Paul Sabatier de Toulouse (France) (Adounvo, 2001). Le zooplancton est récolté par filtration de 10 litres d'eau de chaque bassin au moyen d'un filet à $50 \mu \mathrm{m}$ de maille qui retient la quasi-totalité du zooplancton. Les microorganismes du filtrat sont fixés par ajout de formol à $5 \%$ environ. Un échantillon du filtrat est prélevé au moyen d'une pipette Eppendorf (capacité : $1000 \mu \mathrm{l}$ ), observé au microscope électronique (type MOTIC, G 10X) et systématiquement compté. La densité (D), la production journalière (P) et l'accroissement (Kr) du zooplancton sont calculés pour chaque bassin à partir des formules suivantes :

- $\mathrm{D}=\left(\mathrm{n} / \mathrm{v}_{1}\right) \times\left(\mathrm{v}_{2} / \mathrm{v}_{3}\right)$ avec $\mathrm{n}=$ nombre d'individus comptés, $\mathrm{v}_{1}=$ volume du filtrat prélevé, $\quad \mathrm{v}_{2}=$ volume du filtrat concentré, $\mathrm{v}_{3}=$ volume d'eau filtrée

- $\mathrm{P}=(\mathrm{Nt}-\mathrm{No}) / \mathrm{t}$, avec $\mathrm{No}=$ nombre initial $(\mathrm{mL})$ et $\mathrm{Nt}=$ nombre final $(\mathrm{mL}), \mathrm{t}=$ durée de production.

$-\mathrm{Kr}=(\ln \mathrm{N}-\ln \mathrm{No}) / \mathrm{t}$

Les différentes moyennes sont comparées (au seuil de 5\%) avec le logiciel StatView. A cet effet, l'analyse de variance à un critère de classification (ANOVA 1) (Scherrer, 1984 ; Dagnelie, 1984) et le LSD (Least significant Difference) de Fisher (Saville, 1990) sont utilisés. 


\section{RESULTATS}

Les organismes de la faune planctonique identifiés lors de cette expérimentation sont rassemblés dans 4 groupes à savoir : les rotifères, les cladocères, les copépodes (adultes + copépodites) et les nauplius de copépodes.

\section{Paramètres relevés}

$\mathrm{Au}$ cours de l'expérience, l'évolution de la température n'a pas marqué beaucoup de fluctuations dans les bassins. Les valeurs extrêmes sont de $28,1^{\circ} \mathrm{C}$ et de $31,3^{\circ} \mathrm{C}$ (avec une moyenne de $\left.29,6 \pm 0,1{ }^{\circ} \mathrm{C}\right)$. La concentration moyenne de l'oxygène dissout dans les bassins est de $6,8 \pm 0,7 \mathrm{mg} . \mathrm{L}^{-1}$ et la photopériode est naturelle soit environ 12 heures lumière et 12 heures obscurité. Le pH moyen est de 9,1 $\pm 0,1$. Aucune différence significative de température, de l'oxygène dissous et du $\mathrm{pH}$ n'est observée entre les bassins $(p>0,05)$.

Les valeurs moyennes des paramètres nutritifs (nitrates et phosphates) et biologiques (chlorophylle a et zooplancton) relevés pendant l'expérience sont consignées dans le Tableau 1. Il n'existe aucune différence significative $(p>0,05)$ entre les valeurs moyennes de ces paramètres pour les traitements et leur répétition dans les bassins 1 et 2. Ce qui a permis de considérer les moyennes des deux bassins (bassin test et bassin répétition) dans la suite de l'expression des résultats.

Qualité nutritive des milieux de production : nitrates et phosphates

Les nitrates

Les valeurs des nitrates varient significativement $(\mathrm{p}<0,05)$ de $0,05 \mathrm{mg} . \mathrm{L}^{-1}$ (J0) à $3,9 \mathrm{mg} . \mathrm{L}^{-1}(\mathrm{~J} 2)$ et à $1,05 \mathrm{mg} . \mathrm{L}^{-1}(\mathrm{~J} 23)$ dans les bassins fertilisés à la bouse de vache ; de $0,05 \mathrm{mg} \cdot \mathrm{L}^{-1}(\mathrm{~J} 0)$ à $2,50 \mathrm{mg} \cdot \mathrm{L}^{-1}(\mathrm{~J} 2)$ et à 1,9 mg. $\mathrm{L}^{-1}(\mathrm{~J} 23)$ dans les bassins fertilisés à la fiente de volaille et de $0,05 \mathrm{mg} . \mathrm{L}^{-1}$ (J0) à 2,75 mg. $\mathrm{L}^{-1}(\mathrm{~J} 2)$ et à $1,6 \mathrm{mg} . \mathrm{L}^{-1}(\mathrm{~J} 23)$ dans les bassins témoins. Les valeurs maximales de 4,55 mg.L $\mathrm{L}^{-1}$ (J13) et de 3,9 mg.L $\mathrm{L}^{-1}$ (J15) sont obtenues respectivement dans les bassins fertilisés avec la fiente de volaille et la bouse de vache (Figure 1). Les valeurs moyennes de nitrates obtenues dans les bassins fertilisés à la bouse de vache $\left(2,46 \pm 0,2 \mathrm{mg} . \mathrm{L}^{-1}\right)$ et à la fiente de volaille $\left(2,39 \pm 0,01 \mathrm{mg} . \mathrm{L}^{-1}\right)$ ne présentent aucune différence significative $(\mathrm{p}>$ $0,05)$ entre elles. Mais ces moyennes sont significativement différentes $(p<0,05)$ de celle obtenue dans les bassins témoins qui est de $1,91 \pm 0,08 \mathrm{mg} . \mathrm{L}^{-1}$.

\section{Les phosphates}

Les teneurs en phosphates varient significativement $(\mathrm{p}<0,05)$ de $0,19 \mathrm{mg} \cdot \mathrm{L}^{-1}$ (J0) à $8,58 \mathrm{mg} . \mathrm{L}^{-1}(\mathrm{~J} 2)$ et à $11,64 \mathrm{mg} . \mathrm{L}^{-1}(\mathrm{~J} 23)$ dans les bassins fertilisés à la bouse de vache et de $0,19 \mathrm{mg} . \mathrm{L}^{-1}$ (J0) à 19,79 mg. $\mathrm{L}^{-1}$ (J2) puis à 7,84 mg. $\mathrm{L}^{-1}(\mathrm{~J} 23)$ dans les bassins fertilisés à la fiente de volaille. Dans les bassins témoins, les teneurs en phosphates sont restées faibles tout au long de l'expérimentation et passent de $0,19 \mathrm{mg} . \mathrm{L}^{-1}$ (J0) à $1,33 \mathrm{mg} . \mathrm{L}^{-1}(\mathrm{~J} 2)$ et à $0,57 \mathrm{mg} . \mathrm{L}^{-1}(\mathrm{~J} 23)$ (Figure 2). La quantité moyenne de phosphates obtenue dans les bassins fertilisés à la fiente de volaille $\left(11,74 \pm 0,19 \mathrm{mg} . \mathrm{L}^{-1}\right)$ est significativement $(\mathrm{p}<0,05)$ plus élevée que celles obtenues dans les bassins fertilisés à la bouse de vache $\left(8,12 \pm 0,44 \mathrm{mg} \cdot \mathrm{L}^{-1}\right)$ et les bassins témoins $\left(1,61 \pm 0,54 \mathrm{mg} \cdot \mathrm{L}^{-1}\right)$.

\section{Les paramètres biologiques du phytoplancton}

La biomasse de phytoplancton dans les bassins est évaluée à travers la mesure de la production de chlorophylle-a. Certaines espèces de phytoplancton retrouvées lors des opérations d'identification et de comptage du zooplancton au microscope sont aussi recensées.

Le taux de chlorophylle-a qui est environ de 0,005 mg. $\mathrm{L}^{-1}$ (J0) dans tous les bassins a connu une augmentation significative $(\mathrm{p}<0,05)$ pour atteindre 1,69 mg. $\mathrm{L}^{-1}(\mathrm{~J} 31)$ dans les bassins fertilisés à la bouse de vache et 1,015 mg.L $\mathrm{L}^{-1}$ (J31) dans les bassins fertilisés à la fiente de volaille. Dans les bassins témoins, le taux de chlorophylle-a est resté faible et a varié très peu de 0,005 
mg.L - $^{-1}$ (J0) à 0,045 mg.L ${ }^{-1}$ (J31) (Figure 3). La quantité maximale $\left(2,55\right.$ mg. $\left.\mathrm{L}^{-1}\right)$ de chlorophylle-a est obtenue avec la fiente de volaille au J4. Le taux moyen de chlorophyllea obtenu dans les bassins fertilisés à la fiente de volaille $\left(1,12 \pm 0,6 \quad \mathrm{mg} . \mathrm{L}^{-1}\right) \quad$ est significativement différent $(\mathrm{p}<0,05)$ de ceux obtenus dans les bassins fertilisés à la bouse de vache $\left(0,6 \pm 0,03 \mathrm{mg} . \mathrm{L}^{-1}\right)$ et les bassins témoins $\left(0,04 \pm 0,01 \mathrm{mg} . \mathrm{L}^{-1}\right)$.

Concernant les espèces du phytoplancton, au total 8 espèces sont identifiées. Il s'agit de Chodatella quadriseta, Coelastrum microporum, Pediastrum boryanum, Pediastrum clathratum, Scenedesmus acuminatus, Scenedesmus quadricauda, Tetraspora gelatinosa, Volvox aureus. Parmi ces espèces, 5 sont retrouvées dans les bassins fertilisés à la bouse de vache et à la fiente de volaille et 3 dans les bassins témoins (Tableau 2). L'espèce $S$. quadricauda qui est présente dans les 3 milieux est plus abondante dans les bassins fertilisés à la fiente de volaille que dans les autres bassins.

\section{Les paramètres biologiques du zooplancton \\ La densité}

Les plus fortes densités sont de 3046 ind. $\mathrm{L}^{-1}$ et de 2022 ind. $\mathrm{L}^{-1}$ pour respectivement les bassins à fiente de volaille et à bouse de vache. Les plus faibles densités sont obtenues évidemment dans les bassins témoins (aucun apport de fertilisant) avec 772 ind. $L^{-1}$ comme la moyenne la plus élevée. Tout au long de la période de l'expérimentation, les densités du zooplancton (tous groupes confondus) dans les bassins fertilisés à la fiente de volaille sont plus élevées $(p<0,05)$ que dans les autres bassins (Figure 4). Elles sont suivies des densités obtenues dans les bassins fertilisés à la bouse de vache. Il y a une différence hautement significative entre les densités du zooplancton obtenues avec la fiente de volaille, la bouse de vache et le témoin ( $\mathrm{p}<$ 0,0001).

\section{Production journalière et accroissement du zooplancton}

La production journalière la plus élevée (41 ind. $\mathrm{L}^{-1} . \mathrm{J}^{-1}$ ) est obtenue dans les bassins fertilisés à la fiente de volaille et la plus faible dans les bassins témoins (20 ind. $\left.\mathrm{L}^{-1} . \mathrm{J}^{-1}\right)$ (Figure 5). Cette production journalière des bassins à fiente de volaille est significativement différente $(\mathrm{p}<0,05)$ de celle des bassins à bouse de vache.

Le taux d'accroissement intrinsèque est dans tous les cas supérieur à 0,5 sauf dans les bassins témoins où on a enregistré un taux de 0,3 (Figure 6). Il n'y a pas de différence significative entre les taux d'accroissement du zooplancton des bassins fertilisés à la bouse de vache et à la fiente de volaille $(\mathrm{p}>0,05)$.

Abondance des groupes zoologiques de la faune planctonique dans les bassins

Dans les bassins fertilisés à la fiente de volaille, les cladocères sont les plus abondants $(36,18 \%)$. Après viennent les nauplius de copépodes $(33,34 \%)$, les rotifères $(24,78 \%)$ et les copépodes à l'état copépodites et adultes $(5,70 \%)$. Mais dans les bassins fertilisés à la bouse de vache, ce sont les nauplius de copépodes $(34,01 \%)$ qui sont les plus abondants. Ils sont suivis des rotifères $(27,56 \%)$, des cladocères $(25,83 \%)$ et des copépodes $(12,61 \%)$. De même, dans les bassins non fertilisés, les nauplius de copépodes viennent en tête en représentant $35,36 \%$ des populations. Ils sont suivis des cladocères $(31,74 \%)$, des rotifères $(26,39 \%)$ et des copépodes (adultes + copépodites : 6,51\%) (Figure 7). En général, les copépodes sont relativement plus abondants dans les bassins fertilisés à la bouse de vache que dans les autres bassins.

\section{DISCUSSION}

Les paramètres nutritifs et biologiques

Les taux de nitrates dosés au début de l'expérimentation sont faibles. Les jours suivants, ces taux de nitrates ont augmenté sensiblement dans les milieux et ont varié très 
Tableau 1: Valeurs moyennes des paramètres relevés dans les différents bassins au cours de l'expérience. Les valeurs qui portent la même lettre ne sont pas significativement différentes ( $\mathrm{p}>$ $0,05)$.

\begin{tabular}{|c|c|c|c|c|c|c|c|c|}
\hline \multirow{2}{*}{\multicolumn{2}{|c|}{$\begin{array}{l}\text { Milieux de } \\
\text { production }\end{array}$}} & \multicolumn{7}{|c|}{ Paramètres } \\
\hline & & \multirow{2}{*}{$\begin{array}{c}\begin{array}{c}\mathbf{N O}_{3} \\
(\mathbf{m g} / \mathbf{L})\end{array} \\
2,64^{\mathrm{a}}\end{array}$} & \multirow{2}{*}{$\begin{array}{c}\begin{array}{c}\mathbf{P O}_{4} \\
(\mathbf{m g} / \mathbf{L})\end{array} \\
7,81^{\mathrm{a}}\end{array}$} & \multirow{2}{*}{$\begin{array}{c}\begin{array}{c}\text { Chl-a } \\
(\mathbf{m g} / \mathbf{L})\end{array} \\
0,54^{\mathrm{a}}\end{array}$} & \multirow{2}{*}{$\begin{array}{c}\begin{array}{c}\text { Cla } \\
\text { (ind/L) }\end{array} \\
204,40^{\mathrm{a}}\end{array}$} & \multirow{2}{*}{$\begin{array}{c}\begin{array}{c}\text { Rot } \\
\text { (ind/L) }\end{array} \\
256,32^{\mathrm{a}}\end{array}$} & \multirow{2}{*}{$\begin{array}{l}\begin{array}{l}\text { Nau } \\
\text { (ind/L) }\end{array} \\
281,03^{\mathrm{a}}\end{array}$} & \multirow{2}{*}{$\begin{array}{c}\begin{array}{c}\text { Cop } \\
\text { (ind/L) }\end{array} \\
112,0^{\mathrm{a}}\end{array}$} \\
\hline $\begin{array}{l}\text { Bouse } \\
\text { de }\end{array}$ & Bassin 1 & & & & & & & \\
\hline vache & Bassin 2 & $2,27^{\mathrm{a}}$ & $8,43^{\mathrm{a}}$ & $0,65^{\mathrm{a}}$ & $269,05^{\mathrm{a}}$ & $248,92^{\mathrm{a}}$ & $342,40^{\mathrm{a}}$ & $119,0^{\mathrm{a}}$ \\
\hline $\begin{array}{l}\text { Fiente } \\
\text { de }\end{array}$ & Bassin 1 & $2,40^{\mathrm{a}}$ & $11,60^{\mathrm{b}}$ & $1,12^{b}$ & $492,28^{b}$ & $415,05^{\mathrm{b}}$ & $460,52^{b}$ & $83,91^{\mathrm{b}}$ \\
\hline volaille & Bassin 2 & $2,38^{\mathrm{a}}$ & $11,87^{\mathrm{b}}$ & $1,11^{\mathrm{b}}$ & $570,44^{\mathrm{b}}$ & $312,60^{\mathrm{b}}$ & $518,54^{\mathrm{b}}$ & $83,60^{\mathrm{b}}$ \\
\hline $\begin{array}{l}\text { Témoin } \\
\text { (non }\end{array}$ & Bassin 1 & $1,96^{\mathrm{b}}$ & $1,22^{\mathrm{c}}$ & $0,04^{\mathrm{c}}$ & $80,29^{c}$ & $127,00^{\mathrm{c}}$ & $80,05^{\mathrm{c}}$ & $19,93^{\mathrm{c}}$ \\
\hline fertilisé) & Bassin 2 & $1,85^{\mathrm{b}}$ & $1,19^{\mathrm{c}}$ & $0,04^{\mathrm{c}}$ & $154,65^{\mathrm{c}}$ & $68,40^{\mathrm{c}}$ & $181,64^{\mathrm{c}}$ & $28,24^{\mathrm{c}}$ \\
\hline
\end{tabular}

Tableau 2 : Les espèces de phytoplancton identifiées dans les différents milieux de production.

\begin{tabular}{lccc}
\hline Espèces & Témoin & Fiente de volaille & Bouse de vache \\
\hline Chodatella quadriseta & & + & \\
Coelastrum microporum & + & & \\
Pediastrum boryanum & & ++ & + \\
Pediastrum clathratum & + & ++ \\
Scenedesmus acuminatus & + & +++ & + \\
Scenedesmus quadricauda & + & +++ & + \\
Tetraspora gelatinosa & & & + \\
Volvox aureus & & &
\end{tabular}

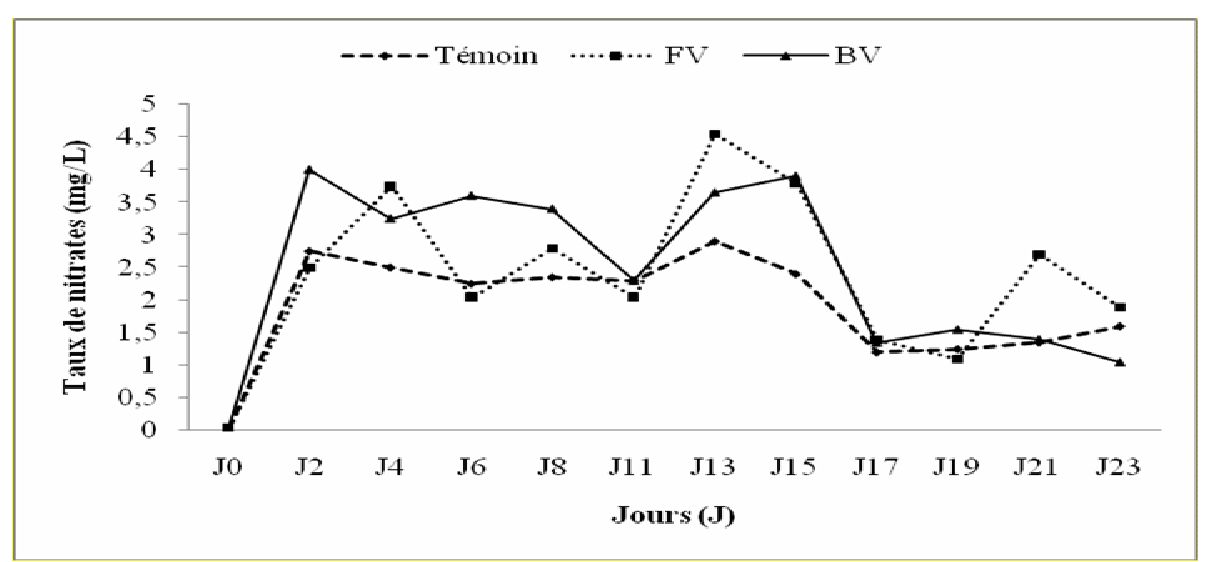

Figure 1: Evolution des taux de nitrates dissous dans les différents bassins au cours de l'expérience. 


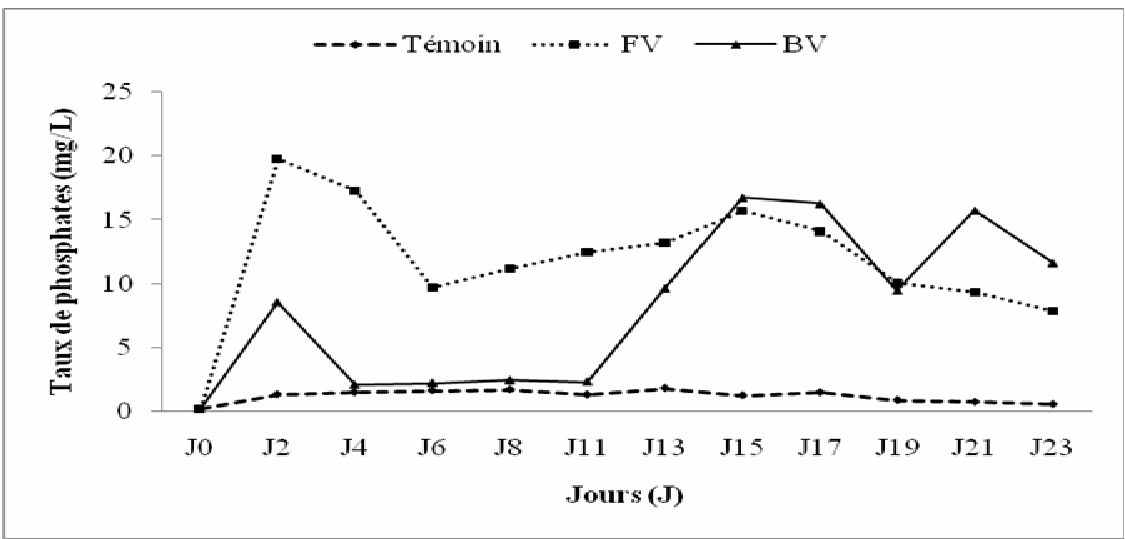

Figure 2 : Evolution des taux de phosphates dans les différents bassins au cours de l'expérience.

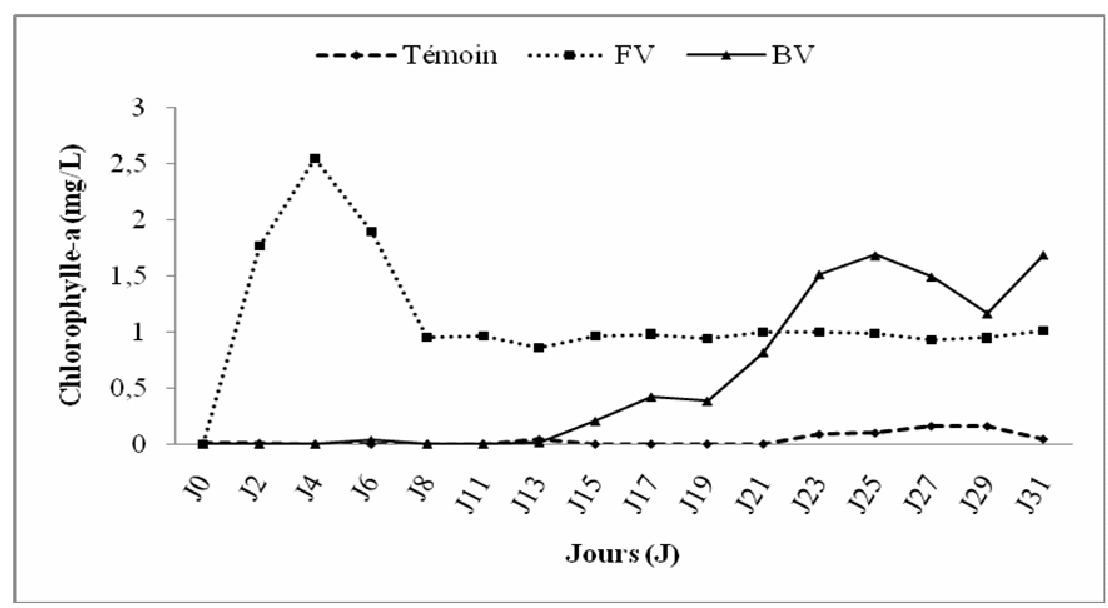

Figure 3: Evolution du taux de chlorophylle-a dans les différents bassins au cours de l'expérience.

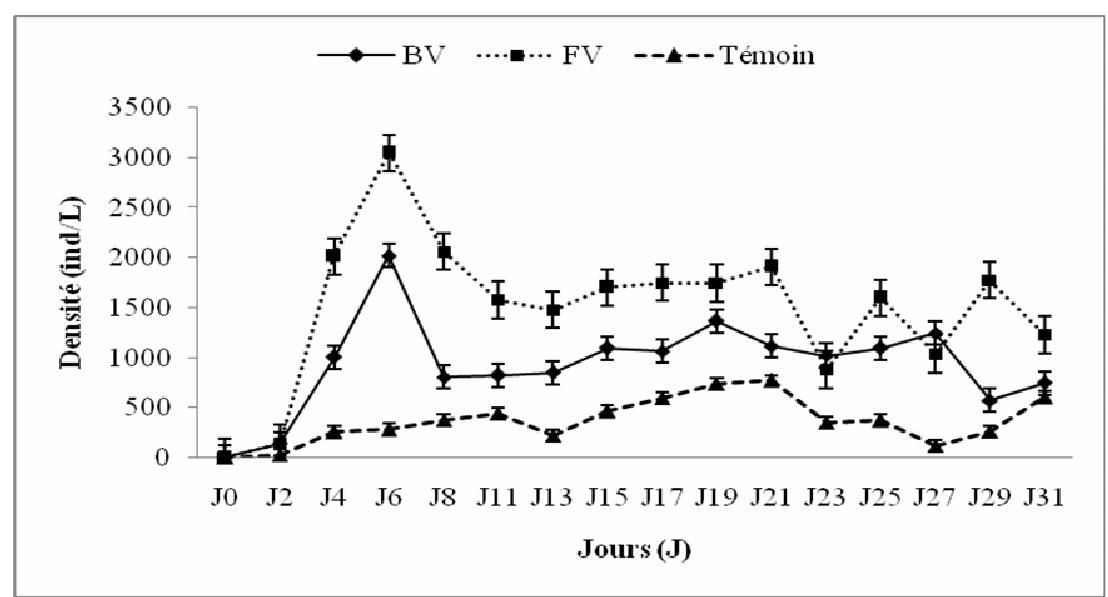

Figure 4: Densités moyennes du zooplancton dans les différents bassins au cours de l'expérience. 


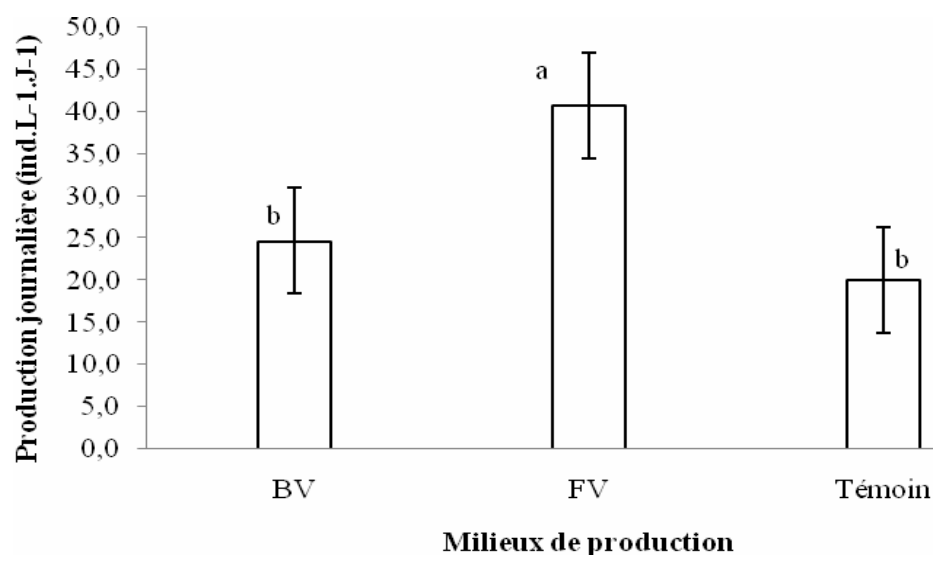

Figure 5: Production journalière du zooplancton dans les différents bassins.

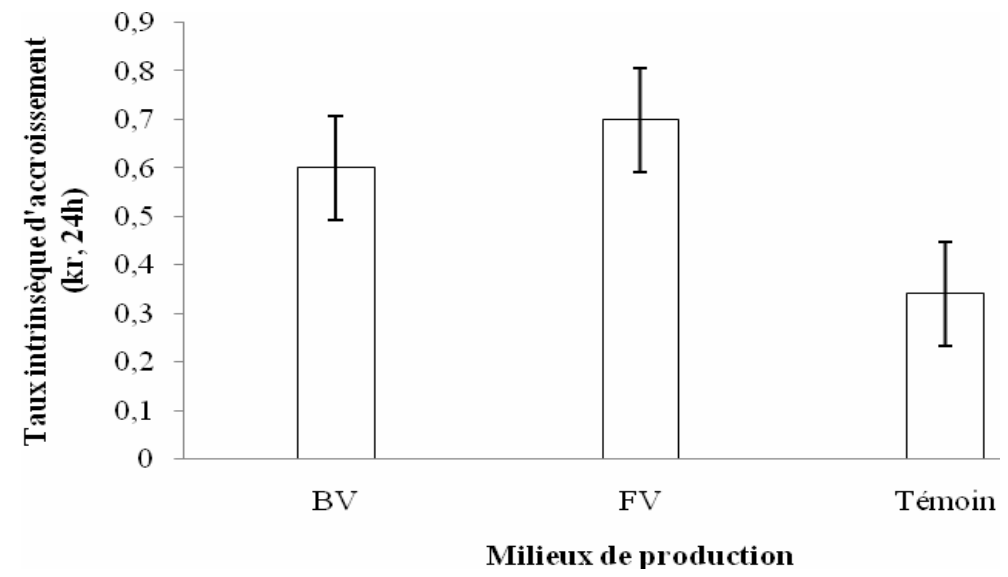

Figure 6 : Taux intrinsèque d'accroissement du zooplancton dans les différents bassins.

peu. Les bassins fertilisés ont donné les fortes valeurs. Il n'y a pas de différence significative entre les valeurs moyennes de nitrates obtenues dans les bassins fertilisés à la bouse de vache et à la fiente de volaille.

Tout comme les nitrates, les phosphates sont faibles au début de l'expérimentation. Dans les bassins témoins, ces taux sont restés faibles pendant toute la période et tendent à s'annuler vers la fin. Par contre dans les bassins fertilisés, les taux de phosphate ont connu une augmentation avec deux pics. Ainsi, très tôt (J2), un premier pic est obtenu dans ces bassins. Mais le pic des bassins à fiente de volaille est situé largement au dessus de celui de bassins à bouse de vache. Après ce premier pic dans les bassins à fiente de volaille, les taux de phosphate varient très peu pour présenter un second pic inférieur au premier au J15. Ceci est dû à la rapidité de la fiente de volaille à se décomposer et à libérer les sels nutritifs dans l'eau. Cette tendance de l'évolution de phosphate dans les bassins à fiente a été déjà observée par Shep (1994) lors d'un élevage monospécifique de Moina micrura, de Diaphanosoma excisum et de Thermocyclops sp en bassins fertilisés à la fiente de volaille. 
FV

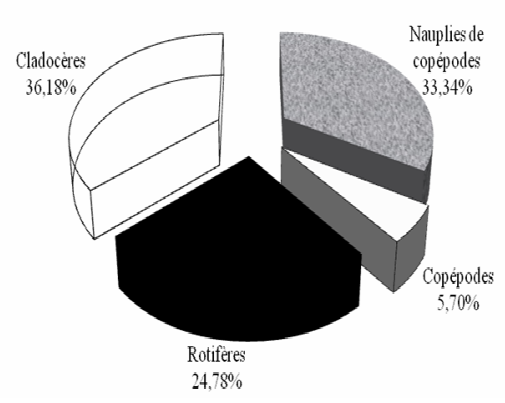

BV

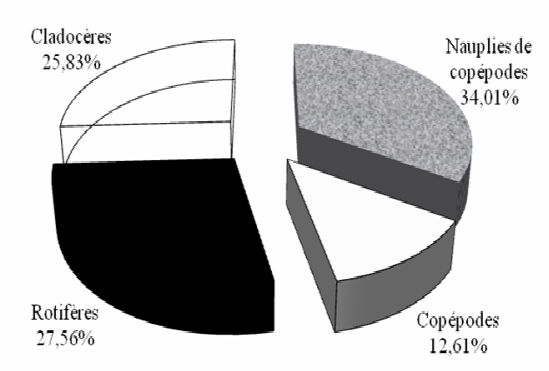

Témoin

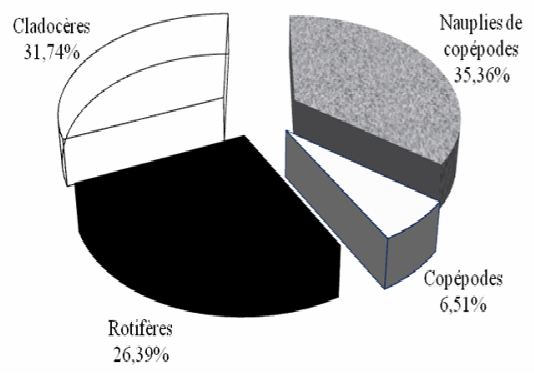

Figure 7: Abondances relatives des groupes zoologiques de la faune planctonique dans différents bassins.

Dans les bassins à bouse de vache, le second pic est supérieur au premier pic. Ce qui est le résultat d'une décomposition relativement lente de la bouse de vache. En effet, la décomposition de la bouse de vache est lente par rapport à la fiente de volaille à cause de sa richesse en cellulose.

Généralement, les taux de phosphate très élevés des bassins à fiente de volaille résultent sans doute de la richesse en phosphore de la fiente de poule (Delincé, 1992). Par ailleurs, les teneurs en nitrates et en phosphates des bassins fertilisés à la fiente de volaille sont comparables voire supérieures à celles obtenues par Sévein-Reyssac (1994) dans les bassins de cultures d'algues et d'élevage de Daphnia avec les lisiers de porc dont les valeurs moyennes se situent entre 2,7 et $7,9 \mathrm{mg} / \mathrm{L}$ pour les nitrates et entre 3,7 et 9,9 $\mathrm{mg} / \mathrm{L}$ pour les phosphates.

L'effet de la fertilisation à la fiente de volaille sur les micro-algues est bien justifié par le développement d'une biomasse phytoplanctonique importante comparativement aux autres milieux. Dans ces bassins fertilisés à la fiente de volaille, le pic de la production phytoplanctonique est atteint en moins d'une semaine; et les micro-algues ont commencé à se multiplier, ceci est confirmé par la forte quantité de chlorophylle-a dans ces bassins. Or dans les bassins fertilisés à la bouse de vache, ce n'est qu'environ deux semaines après la fertilisation que le "bloom" phytoplanctonique s'est installé. La valeur de chlorophylle-a obtenue dans les bassins fertilisés à la fiente de volaille est supérieure à celles obtenues dans les autres bassins $(\mathrm{p}<$ $0,05)$.

Cependant, dans tous les milieux, les quantités de chlorophylle-a restent relativement faibles (moyenne $<2 \mathrm{mg} / \mathrm{L}$; Canovas et al., 1991). Ce qui pose le problème de quantité optimale de fiente à utiliser pour une bonne production de micro-algues dont 
dépendent les organismes du zooplancton. Sur le plan qualitatif, les bassins fertilisés à la fiente de volaille sont aussi riches en espèces phytoplanctoniques que les bassins fertilisés à la bouse de vache. Néanmoins, l'espèce commune aux trois milieux (S. quadricauda) est plus abondante dans les bassins à fiente de volaille.

\section{La production du zooplancton}

Durant toute l'expérimentation, la production du zooplancton dans les bassins fertilisés est largement supérieure à celle des bassins non fertilisés (témoins); et la différence est hautement significative ( $p$ < $0,05)$. Cette différence serait liée à la richesse en micro-algues des milieux fertilisés.

Les bassins à fiente de volaille ont donné les plus fortes densités du zooplancton avec la meilleure production journalière ( $\mathrm{p}<$ $0,05)$ et la fiente se révèle comme le meilleur fertilisant. C'est la conséquence de sa richesse algale plus importante que dans les autres milieux. Car, selon Seyer (2002), la multiplication du zooplacton augmente dès lors qu'ils trouvent dans leur milieu une abondante nourriture algale. Les densités de production sont comprises entre 148,16 ind.L 1 et 3045,53 ind. $\mathrm{L}^{-1}$ (moyenne $=1597,27$ ind. $\mathrm{L}^{-1}$ ) dans les bassins à fiente de volaille ; 140,34 ind. $\mathrm{L}^{-1}$ et 2021,93 ind. $\mathrm{L}^{-1}$ (moyenne = 999,42 ind. $\mathrm{L}^{-1}$ ) pour les bassins à bouse de vache. Cette densité moyenne obtenue avec la fiente de volaille est située dans la gamme de densités de plancton des lacs eutrophes (densités comprises entre 1000 et 2000 ind. $\mathrm{L}^{-1}$ ) (Pourriot, 1975).

L'abondance relative des cladocères, des rotifères et des nauplies de copépodes (tous herbivores; Dussart, 1967 ; Pourriot et al., 1982 ; Bonou, 1990 ; Gliwicz, 1994) dans la fiente de volaille témoigne la richesse phytoplanctonique de ce milieu. Cette assertion rejoint celle de Margalef (1983), Blancher (1984), Orcutt et Pace (1984), Pace (1986) et de Zébazé Togouet et al. (2004) qui soulignent que la forte représentativité des rotifères dans un milieu indique biologiquement le niveau trophique élevé de ce milieu.

Le taux d'accroissement intrinsèque du zooplancton est plus élevé dans les milieux fertilisés $(\mathrm{p}<0,05)$. La valeur maximale de 0,7 obtenue avec la fiente de volaille serait liée à l'abondance des espèces de cladocères et de rotifères qui présentent souvent des taux d'accroissement élevés. Car, le taux d'accroissement d'une espèce de cladocère (Moina micrura) peut atteindre 0,9 (Bonou, 1990) et plus (Shep, 1994). Celui de Brachionus calyciflorus (rotifère) élevée sur une culture d'algue Dictyosphaerium chlorelloides est de 0,53 (Awaïss et Kestemont, 1997). Dans les étangs de pisciculture de Layo, les copépodes présentent un taux d'accroissement intrinsèque de l'ordre de 0,42 et 0,66 (Bonou, 1990). Ainsi, les capacités de développement et les profils d'évolution du zooplancton diffèrent selon les espèces (Shep, 1994) et le taux d'accroissement intrinsèque en élevage plurispécifique dépend de celui de l'espèce dominante.

En conclusion, les milieux fertilisés donnent les meilleures productions du zooplancton. Cette production est plus élevée avec la fiente de volaille qui offre au zooplancton les bonnes conditions nutritives. Ainsi, la fiente de volaille se révèle comme le meilleur fertilisant pour la production du zooplancton. Cependant, la quantité de fiente à utiliser pour une condition optimale de production du zooplancton reste à déterminer afin d'éviter soit la pollution du milieu de production, soit la médiocrité des conditions nutritives.

\section{REMERCIEMENTS}

Monsieur Hyppolite AGADJIHOUEDE est doctorant en Sciences Agronomiques à l'Université d'Abomey-Calavi, Bénin, et boursier de la Coopération Allemande dans le cadre du projet BIOTA WEST.

\section{REFERENCES BIBLIOGRAPHIQUES}

Adounvo DU. 2001. Etude comparée de la productivité primaire des lacs Nokoué et 
Ahémé au Bénin. Mémoire d'Ingénieur Agronome, Université d'Abomey-Calavi, p. 98 .

Awaïss A, Kestemont P, Micha J-C. 1993. Etude du premier alevinage du poissonchat africain, Clarias gariepinus (Birchell, 1822), avec le rotifère d'eau douce, Brachionus calyciflorus (Pallas). EAS Spec. Publ., 18 : 443-453.

Awaïss A, Kestemont P. 1997. Dynamique de production et qualité nutritionnelle du rotifère d'eau douce Brachionus Calyciflorus. Aquat. Living Resour., 10: 111-120.

Barnabé G. 1991. Bases Biologiques et Ecologiques de l'Aquaculture. Ed. LAVOISIE. TEC et DOC; 500.

Blancher EC. 1984. Zooplankton-trophic state relationships in some north and central Florida lakes. Hydrobiologia, 109: 251263.

Bonou CA. 1990. Etude de la productivité planctonique dans les étangs d'aquaculture en milieu saumâtre tropical. Thèse de Doctorat de l'Institut National Polytechnique de Toulouse, p. 227.

Bonou CA, Pagano M, Saint-Jean L. 1991. Développement et croissance en poids de Moina (cf) micrura et de Mesocyclops ogunnus dans un milieu saumâtre tropical: les étangs de pisciculture de Layo (Côte-d'Ivoire). Rev. Hydrobio. Trop., 24 : 287-303.

Broussard MCJR, Reyes R, Raguindin F. 1983. Evaluation of hatchery management Tilapia nilotica at two densities with fertilization and supplemental feeding. 1ère partie. Aquarevue, 11: 35 -39.

Canovas S, Casellas C, Picot B, Pena G, Bontoux J. 1991. Evolution annuelle du peuplement zooplanctonique dans un lagunage à haut rendement et incidence du temps de séjour. Rev. Sci. Eau, 4: 269289.

Dabbadié L. 1996. Etude de la viabilité d'une pisciculture rurale à faible niveau d'intrant dans le Centre-Ouest de la Côte
d'Ivoire : approche du réseau trophique. Thèse de doctorat de l'Université de Paris 6 en Océanologie biologique, p. 207.

Dagnelie P. 1984. Théorie et Méthodes Statistiques. Applications Agronomiques. Tome II. Les Presses Agronomiques de Gembloux : Gembloux ; 463.

Delincé G. 1992. The Ecolohy of the Fish Pond Ecosystem with Special Reference to Africa. Kluwer Academic Publishers: Amsterdam; 230.

Duncan A, Gulati RD. 1981. Parakrama Samudra (Sri Lanka) projet - a study of a tropical lake ecosystem, 3. Composition, density and distribution of the zooplankton in 1979. Verh. Int. Ver. Theor. Angew. Limnol., 21 : 1001-1008.

Dussart B. 1967. Les Copépodes des Eaux Usées d'Europe Occidentale. Thome I: Calanoïdes et Harpacticö̈des. Collection 'Faunes et Flores actuelles'. N. Boubée et Cie (Ed.): Paris; 500.

Gliwicz, ZM. 1994. Retarded growth of cladoceran zooplankton in the presence of a copepod predator. Ecologia, 97: 458461.

James CM, Rezeq TA. 1989. Intensive rotifer cultures using chemostats. Hydrobiologia, 186(187): 423-430.

Lorenzen CJ. 1967. Determination of chlorophyll and pheopigments: Spectrophotometric equations. Limnol. Oceanogr., 12: 343-346.

Munawar M, Munawar IF Sprules WG. 1991. The plankton ecology of Lake St. Clair, 1984. In Munawar M Edsall T. (eds), Environmental Assessment and Habitat Evaluation of the Upper Great Lakes Connecting Channels. Hydrobiologia, 219: 203-227.

Orcutt JRD, Pace ML. 1984. Seasonal dynamics of rotifer and crustacean zooplankton populations in a eutrophic, monomitic lake with a note on rotifer sampling techniques. Hydrobiologia, 119: 73-80.

Pace ML. 1986. An empirical analysis of zooplankton community size structure 
across lake trophic gradients. Limnol. Oceanogr., 31: 45-55.

Pourriot R. 1975. Relations prédateur-proie : relations adaptatives et fluctuations des populations $\mathrm{du}$ zooplancton sous l'influence d'une prédation sélective. Ann. Biol., 14(1/2) : 69-85.

Pourriot R, Capblancq J, Champ P, Meyer JA. 1982. Ecologie du Plancton des Eaux Continentales. Collection Ecol. Appl. Sci. Env., Masson (Ed.), 16 ; 198.

Rukera Tabaro S, Micha J-C, Ducarme C. 2005. Technique de production intensive $\mathrm{du}$ poisson-chat africain Clarias gariepinus. Tropicultura, 21(14): 189198.

Saville DJ. 1990. Multiple comparaison procedures: the pratical solution. American Statistician, 44 (2) : 174-180.

Scherrer B. 1984. Biostatistique. Gaëtan Morin: Québec ; 850.

Sévrin-Reyssac J, Combres C, Laux S, Texier C. 1994. Application des techniques du lagunage au récyclage du lisier de porc. Journées Rech. Porcine en France, 26: 123-144.

Seyer J. 2002. Le chant de l'eau. Production de proies vivantes Brachionus plicatilis et
Artemia salina. Ifremer, station Merea, 83 84.

Shep H. 1994. Comparaison des capacities de développement et des processus de régulation en élevage monospécifique de triostypes de proies planctoniques d'intérêt aquacole: Moina micrura, Diaphanosoma excisum et Thermocyclops sp. Mémoire de DEA en Ecologie tropicale, Université Nationale de Côte-d'Ivoire, p. 55.

Vincke HH, Philippart JC. 1984. Mission d'évaluation de la pisciculture en République Populaire du Bénin. Université de Liège (ULg), CECODEL, p. 128.

Zébazé Togouet SH, Njiné T, Kemka N, Nola M, Foto Menbohan S, Monkiedje A, Niyitegeka D, Sime-Ngando T, Jugnia LB. 2004. Variations spatiales et temporelles de la richesse et de l'abondance des rotifères (Brachionidae et Trichonidae) et des cladocères dans un petit lac artificiel eutrophe situé en zone tropicale. Rev. Sci. Eau, 18(4): 485-505. 